\title{
Demonstrating Dothraki: Tracking Tangibles Atop Tabletops Through De-Bruijn Tori
}

\author{
Dennis Schüsselbauer \\ University of Regensburg \\ Regensburg, Germany \\ dennisschuesselbauer@web.de
}

\author{
Andreas Schmid \\ University of Regensburg \\ Regensburg, Germany \\ andreas.schmid@ur.de
}

\author{
Raphael Wimmer \\ University of Regensburg \\ Regensburg, Germany \\ raphael.wimmer@ur.de
}

\begin{abstract}
We demonstrate usage examples and technical properties of Dothraki, an inside-out tracking technique for tangibles on flat surfaces. An optical mouse sensor embedded in the tangible captures a small $(36 \times 36$ pixel $/ 1 \times 1 \mathrm{~mm})$, unique section of a black-and-white De-Bruijn dot pattern printed on the surface. Our system efficiently searches the pattern space in order to determine the precise location of the tangible with sub-millimeter accuracy. Our proof-of-concept implementation offers a recognition rate of up to $95 \%$, robust error detection, an update rate of $14 \mathrm{~Hz}$, and a low-latency relative tracking mode. The MuC demonstration encompasses four separate demos that showcase typical application scenarios and features: a magic lens, two tangibles that know each others relative position, a simple geometry application that measures distances and angles, and tangibles that know on which of multiple surfaces they have been placed.
\end{abstract}

\section{CCS CONCEPTS}

- Human-centered computing $\rightarrow$ Interaction devices; Mixed / augmented reality.

\section{KEYWORDS}

tracking, tangibles, de-bruijn torus, hardware

\section{ACM Reference Format:}

Dennis Schüsselbauer, Andreas Schmid, and Raphael Wimmer. 2021. Demonstrating Dothraki: Tracking Tangibles Atop Tabletops Through De-Bruijn Tori. In Mensch und Computer 2021 (MuC '21), September 5-8, 2021, Ingolstadt. ACM, New York, NY, USA, 3 pages. https://doi.org/10.1145/3473856.3474030

\section{INTRODUCTION: SPATIALLY AWARE TANGIBLES}

Tangibles, also called tangible blocks, or bricks [1] are small, graspable objects that can be used as input devices or as physical representations of digital data and relations [2]. For many applications, it is desirable to determine and track the location or orientation of a tangible. Fitzmaurice [1] calls this capability "spatial However, robust tracking of small, handheld objects is non-trivial. With Dothraki [3] (Figure 1), we presented an approach for tracking the positions of multiple active tangibles on a flat surface that is

This work is licensed under a Creative Commons Attribution International 4.0 License.

MuC '21, September 5-8, 2021, Ingolstadt

(c) 2021 Copyright held by the owner/author(s).

ACM ISBN 978-1-4503-8645-6/21/09.

https://doi.org/10.1145/3473856.3474030

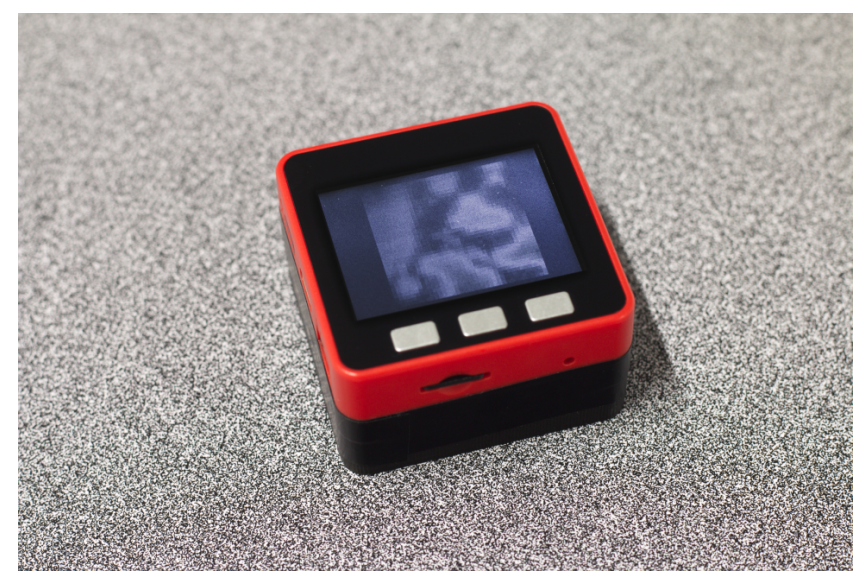

Figure 1: Dothraki is a reference implementation for tracking tangibles on tabletop surfaces. Via an optical mouse sensor, a tangible detects its position within a printed DeBruijn-torus pattern and tracks its own movement with low latency.

covered with a custom dot pattern, a De-Bruijn torus (DBT). An optical sensor from a computer mouse is embedded into the tangible and continuously captures a section of this pattern right under the tangible. The captured image is then used to determine the tangible's location within the pattern space, and therefore within the surface area. Dothraki is low-cost and can be integrated with common microcontroller platforms. It requires no external infrastructure besides a sheet of paper printed with the pattern and a computer that runs the position-tracking code. Relative motion can be tracked with very low latency by the mouse sensor itself. This makes Dothraki suitable for ad-hoc, collaborative applications, such as educational games.

Mensch und Computer 2021 will be the first conference where Dothraki is presented live. This demo paper is based on the full paper and reuses some text and figures.

\section{IMPLEMENTATION}

As the basis for our tangible blocks, we use M5Stack blocks ${ }^{1}$ (Figure 2). For determining the location of the tangible on the surface, we use a PixArt PMW3360 mouse sensor which contains an optical sensor and a laser diode for illumination of the surface. The sensor is attached to the ESP32 via SPI.

Dothraki is able to determine the location of the tangible by instructing the mouse sensor to capture an image of the surface beneath the

\footnotetext{
${ }^{1}$ https://m5stack.com/
} 

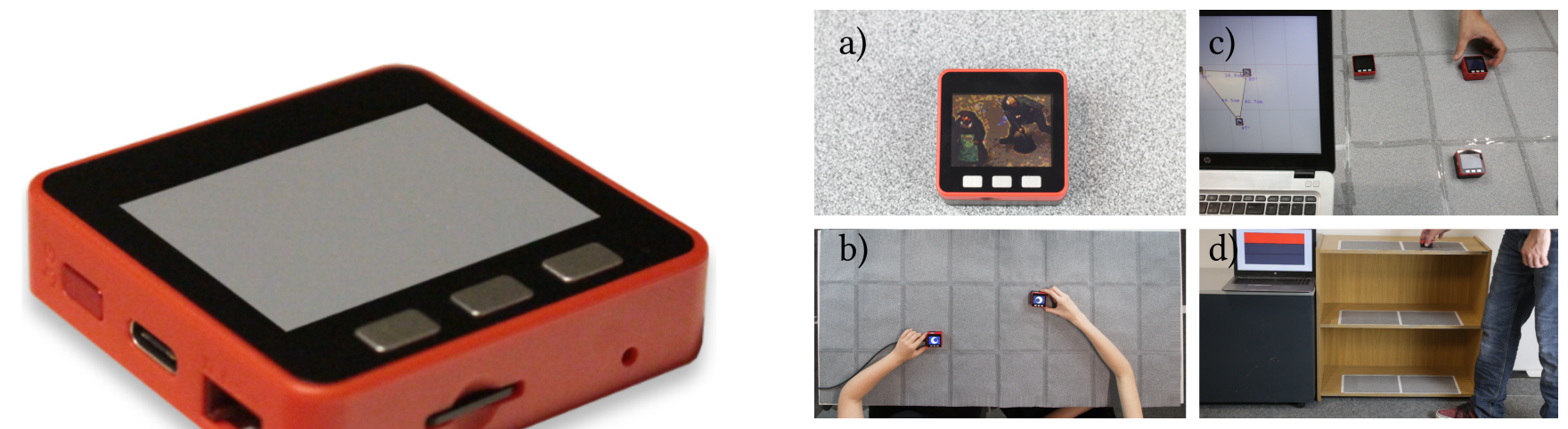

Figure 3: Example applications: a) Magic Lens that allows for scrolling through a virtual image by moving the tangible, $b$ ) Googly Eyes which always look at each other even if the tangibles are picked up and placed elsewhere, c) Distance and Angle Measurements between multiple tangibles, and d) Location Detection where tangibles are tracked across separate surfaces.

and reads movement data directly from the PMW3360 until an answer from the host computer arrives.

This positions Dothraki as a scalable, low-cost alternative to capacitive or optical interactive tabletops. Dothraki can be deployed within minutes and takes up little space when not in use. Therefore, it is uniquely suited for use in classrooms, boardgames, or as an addition to traditional physical desktops. Unlike capacitive and optical tabletops, Dothraki cannot track arbitrary rotation, however.

\section{DEMO APPLICATIONS}

While we see Dothraki as a generic sensing technique that can be embedded in a multitude of applications, we will present four concrete use cases for it. Examples are partially based on demo applications we developed for an earlier prototype that did not yet have DBT tracking integrated $[4,5]$.

The Magic Lens (Figure 3a) allows the user to place the tangible anywhere on the surface and see a section of a larger image on the tangible's screen. By moving the tangible around the surface, the user can move the section of the image that is shown on the screen. As these relative movements are directly tracked by the mouse sensor, latency is low. Such a magic lens could be used for adding digital content to a printed map or image, hiding digital hints on the pages of a physical books, or using the tangible as a cursor for selecting objects projected onto a tabletop.

Mutual Awareness allows multiple tangibles to interact with each other (Figure 3b). In this application, the host computer not only calculates each tangible's position but also transmits the position to all connected tangibles, allowing them to react to each other. In our example, each of the two tangibles displays a stylized eyeball on its screen. The eyeballs always look at each other even if the tangibles are moved around the surface, lifted and put somewhere else, or rotated by multiples of 90 degrees. Mutually aware tangibles can be used as avatars in board games, bimanual input devices, or as building blocks in educational applications where students e.g., have to arrange tangibles in a certain order. 
Distance and Angle Measurements allow for using multiple tangibles to measure physical and mathematical properties (Figure 3c). When two tangibles are placed on the tabletop, the system displays the distance between both on a separate screen. When three tangibles are on the tabletop, the system displays angles and distances between them. This feature can be used to create interactive tangible learning experiences or to implement physical controls for e.g. construction planning.

Location Detection shows that Dothraki can also be used for detecting on which of multiple surfaces in a room the tangible has been placed (Figure 3d). Depending on the available table setup at the venue, attendees will be able to place a tangible at one of multiple tables or locations within a shelf. The system then highlights the chosen location. Such functionality might be used as a foundation for collaborative games within a classroom where students need to place tangibles at certain locations or in certain combinations.

\section{ACKNOWLEDGMENTS}

This project is funded by the Bavarian State Ministry of Science and the Arts and coordinated by the Bavarian Research Institute for Digital Transformation (bidt).

\section{REFERENCES}

[1] George W. Fitzmaurice, Hiroshi Ishii, and William A. S. Buxton. 1995. Bricks: Laying the Foundations for Graspable User Interfaces. In Proceedings of the SIGCHI Conference on Human Factors in Computing Systems (CHI '95). ACM Press/AddisonWesley Publishing Co., New York, NY, USA, 442-449. https://doi.org/10.1145/ 223904.223964

[2] Hiroshi Ishii and Brygg Ullmer. 1997. Tangible Bits: Towards Seamless Interfaces Between People, Bits and Atoms. In Proceedings of the ACM SIGCHI Conference on Human Factors in Computing Systems (CHI '97). ACM Press, Atlanta, Georgia, United States, 234-241. https://doi.org/10.1145/258549.258715

[3] Dennis Schüsselbauer, Andreas Schmid, and Raphael Wimmer. 2021. Dothraki: Tracking Tangibles Atop Tabletops Through De-Bruijn Tori. In Proceedings of the Fifteenth International Conference on Tangible, Embedded, and Embodied Interaction (TEI '21). Association for Computing Machinery, New York, NY, USA, 1-10. https: //doi.org/10.1145/3430524.3440656

[4] Dennis Schüsselbauer, Andreas Schmid, Raphael Wimmer, and Laurin Muth. 2018. DotTrack: absolute und relative Ortsbestimmung von Tangibles mittels eines Maussensors. Gesellschaft für Informatik e.V. (2018). https://doi.org/10.18420/ muc2018-ws05-0500

[5] Dennis Schüsselbauer, Andreas Schmid, Raphael Wimmer, and Laurin Muth. 2018. Spatially-Aware Tangibles Using Mouse Sensors. In Proceedings of the Symposium on Spatial User Interaction (SUI '18). ACM, New York, NY, USA, 173-173. https: //doi.org/10.1145/3267782.3274690 